\title{
Exact Equation of Ellipse Perimeter by Analytical Method
}

\author{
Abolfazl Soltani ${ }^{*}$ \\ Department of Physics, University of Birjand, Birjand, Iran \\ Email: soltani.a.physics@gmail.com
}

\begin{abstract}
As you know, we do not have any exact equation for calculating the perimeter of an ellipse. In this article, we obtain this equation analytically. In this article we will find a way for derivation of the solution of Elliptic integral in terms of elementary functions.
\end{abstract}

Keywords: Ellipse perimeter, Ellipse, Perimeter, Elliptic integral

\section{Introduction}

Calculating the perimeter of an ellipse is difficult analytically, and for more than 300 years all efforts in this direction have failed. To calculate the perimeter of an ellipse using the formula:

$$
r=\frac{a_{0}\left(1-\epsilon_{0}^{2}\right)}{1-\epsilon_{0} \operatorname{Cos} \theta}
$$

which is equation of an ellipse in polar coordinates [1][2], we must calculate the following integral (which is the equation of the length of a polar curve) [1][3].

$$
L=\int_{\alpha}^{\beta} \sqrt{r^{2}+\left(\frac{d r}{d \theta}\right)^{2}} d \theta
$$

Substituting 1 in 2 leads us to integral 3, which is clearly difficult to calculate.

$$
P_{\text {Ellipse }}=a_{0}\left(1-\epsilon_{0}^{2}\right) \int_{0}^{2 \pi} \sqrt{\frac{\left(1-\epsilon_{0} \operatorname{Cos} \theta\right)^{2}+\epsilon_{0}^{2} \operatorname{Sin}^{2} \theta}{\left(1-\epsilon_{0} \operatorname{Cos} \theta\right)^{4}}} d \theta
$$

Therefore, to extract the perimeter of an ellipse, analytically, we must look for another method. 'we define the ellipse as a linear transformation of the circle with radius 1',[4]. This was the sentence that led me to try to extract the perimeter of an ellipse using the perimeter of a circle. If $x$ and $y$ are two points on an ellipse. In such a case, it can be shown, by linear transformation method, that for $\mathrm{b}$ and $\mathrm{a}, y / b$ and $x / a$ are two points on a circle with the following equation [4]:

$$
\left(\frac{x}{a}\right)^{2}+\left(\frac{y}{b}\right)^{2}=1
$$

Familiarity with this subject lead me to think that instead of calculating the perimeter of the ellipse, we can obtain the perimeter of its equivalent or associated circle.

Consider Figure 1. In this figure, you see an ellipse with dimensions a and b, which is surrounded in circle $C_{1}$ with center $O$ and radius $r=a$. From points $A$ and $B$ we have drawn four tangent lines to the ellipse and the parallelogram $A C B D$ was formed. now we can draw a circle with the center $O$ that is tangent to all four faces of this parallelogram. The red circle in 
Fig. 1 is this circle. We call this circle the equivalent circle or the associated circle of ellipse. In this article, we show that the perimeter of our ellipse is equal to the perimeter of the red circle and the formula for the perimeter of ellipse is as follows:

$$
P_{\text {Ellipse }}=\frac{2 \pi a_{0}}{\sqrt{2}^{\epsilon_{0}^{2}}}
$$

Where $a_{0}$ is semi-major axis and $\epsilon_{0}$ is eccentricity of ellipse. This is the equation we have been looking for, at least since Leonhard Euler.

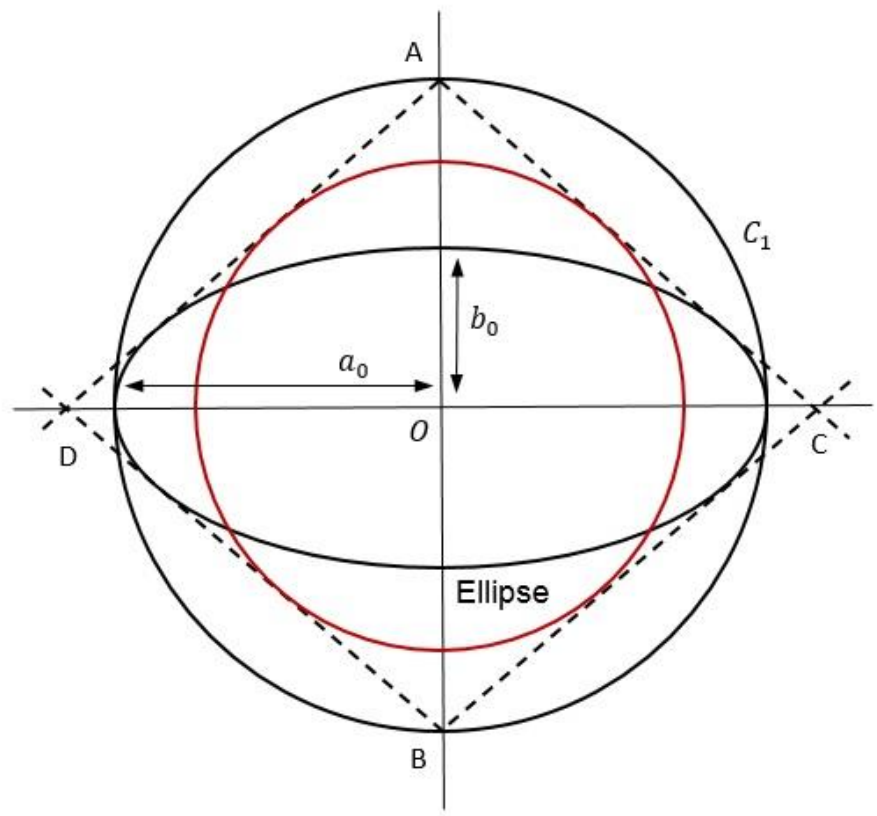

Fig. 1.

\section{Associated Circle of Ellipse}

Using Figure 1, the equation of radius of red circle (associated circle with ellipse) can be obtained by the boundary conditions method. And then obtained the perimeter of the circle. There are two boundary conditions here: 1- The first boundary condition is that in Figure 1, as the $\mathrm{b}$ increases, the ellipse becomes larger, and as a result, the red circle also enlarges, and in the case of $b=a$, the ellipse becomes the red circle:

$$
\begin{gathered}
b_{0}=a_{0} \text { and } b_{0}=a_{0} \sqrt{1-\epsilon_{0}^{2}} \Rightarrow \epsilon_{0}=0 \\
r=\frac{a_{0}\left(1-\epsilon_{0}^{2}\right)}{1-\epsilon_{0} \operatorname{Cos} \theta} \stackrel{(\mathbf{I})}{\Rightarrow} r=a_{0}
\end{gathered}
$$

which it means that the red circle in this case is circle $C_{1}$. In fact, the circle $C_{1}$ is the limit state of the red circle (upper bound). In the case of $b_{0}=a_{0}$ our ellipse is a circle $\left(C_{1}\right)$ and the equation for the perimeter of ellipse is in the form of equation of perimeter of circle namely:

$$
P=2 \pi a_{0}
$$


Therefore, we must obtain the shape of final equation for the perimeter of ellipse in such a way that in the boundary state $\epsilon_{0}=0$ gives us the perimeter of its associated circle namely $2 \pi a_{0}$.

2- The second boundary condition is that in Figure 1 , if the value of $b$ decreases and approaches zero $(b \rightarrow 0)$, in this case our ellipse will be almost in the form of a straight line (mode $b=0$ is not acceptable and gives us an exact straight line that a straight line is never considered an ellipse). In this case, the parallelogram $A C B D$ in Figure 1, which as we have said is a parallelogram tangent to the ellipse, turns into an orange square in Figure 2, which intersects the circle $C_{1}$ at four points. In such a case, the associated circle of ellipse is the green circle which is tangent to the four sides of the orange square (the green circle is the lower boundary of red circle).

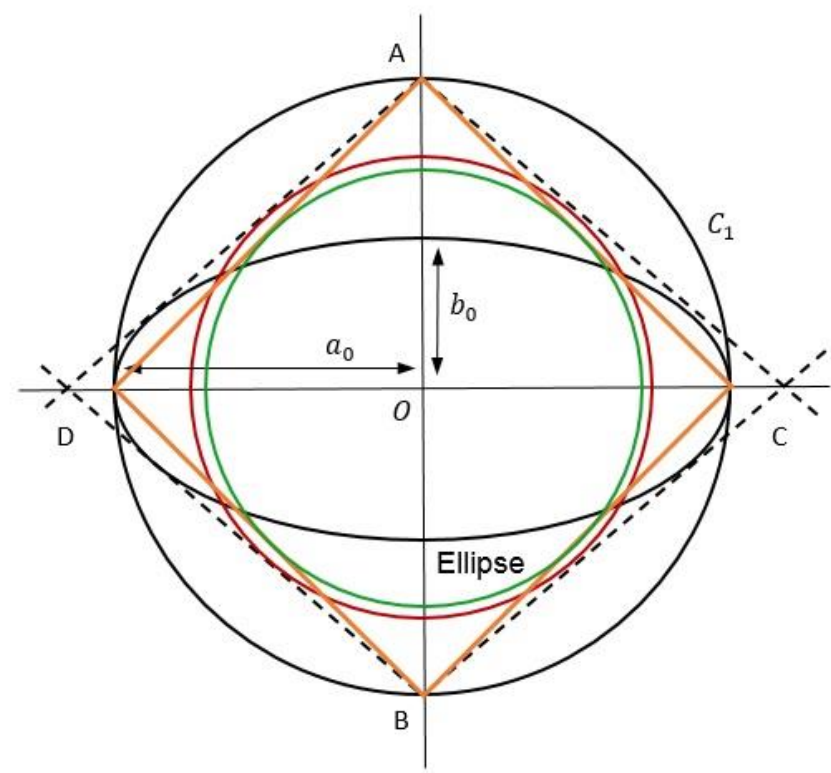

Fig. 2.

Since in boundary condition 1 our ellipse perimeter was equal to the perimeter of its associated circle namely the circle $C_{1}$ (as we said, the red circle in figure 1 , when $r_{\text {red circle }}=a_{0}$, is the circle $C_{1}$ ) in this case also we expect the perimeter of our ellipse be equal to the perimeter of its associated circle namely the green circle. The radius of the green circle according to figure 3 is equal to:

$$
O E^{2}+E H^{2}=a_{0}^{2} \stackrel{O E=E H}{=} 2(O E)^{2}=a_{0}^{2} \Longrightarrow O E=r_{\text {green circle }}=\frac{a_{0}}{\sqrt{2}}
$$

Therefore, the perimeter of green circle is equal to:

$$
P_{\text {green circle }}=2 \pi \frac{a_{0}}{\sqrt{2}}
$$




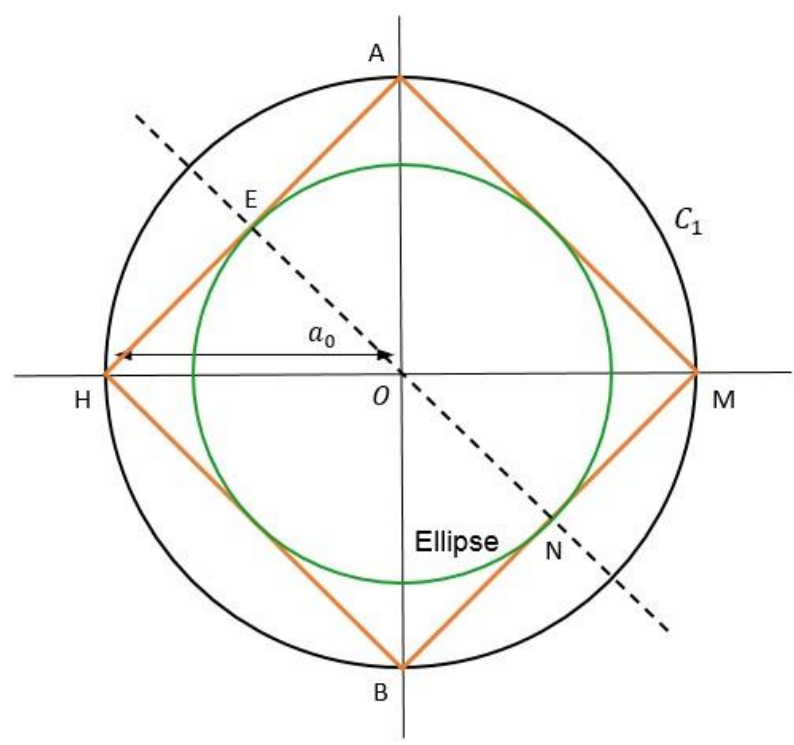

Fig. 3. When $b_{0}$ approaches zero in ellipse in figure 1, ellipse becomes almost a straight line which is almost coincident with the $M H$ line, and its associated circle is the green circle.

So, in the case of $b \rightarrow 0$ or $\epsilon_{0} \rightarrow 1$, the shape of final equation of our ellipse perimeter must be converted to the equation:

$$
P_{\text {ellipse }}=P_{\text {green circle }}=2 \pi \frac{a_{0}}{\sqrt{2}}
$$

Given that we expect the ellipse perimeter equation to contain $a$ and $\epsilon$, applying two boundary conditions 1 and 2 (equations 5 and 6) leads us to the following equation:

$$
P_{\text {Ellipse }}=P_{\text {red circle }}=\frac{2 \pi a_{0}}{\sqrt{2}^{\epsilon_{0}{ }^{m}}}
$$

Where $m$ is a positive Rational number such as $1,2,3, \frac{1}{2}, \frac{1}{3}, \frac{1}{4}$ and etc. The equation 7 is the final shape of equation of ellipse perimeter. In the next section we will obtain the value $m$.

If we substitute $\epsilon=0$ in equation 7, we get equation 5, and if we substitute $\epsilon \rightarrow 1$ in equation 7 , we get equation 6 .

\section{Extract value of $\boldsymbol{m}$}

In this section we use an example and obtain value $m$ by comparing the result of our analytical equation with the Maclaurin equation (the Maclaurin equation is a series with infinite number of sentences that gives us the exact value of the ellipse perimeter).

Consider an ellipse with $a_{0}=10 \mathrm{inch}$ and $b_{0}=5 \mathrm{inch}$. Therefore: $\epsilon_{0}=0.866$. If we consider $m=2$ in such a case we have:

$$
P_{\text {Analytic }}=\frac{2 \pi a_{0}}{\sqrt{2}^{\epsilon_{0}{ }^{m}}}=\frac{20 \pi}{\sqrt{2}^{(0.866)^{2}}}=48.47 \mathrm{inch}
$$


Now we calculate the perimeter of the ellipse with Maclaurin series: The Maclaurin series equation is [5][6]:

$$
P_{M a c}=2 \pi a_{0} \sum_{n=0}^{\infty}\left\{\left(\frac{-1}{2 n-1}\right)\left[\frac{2 n !}{\left(2^{n} n !\right)^{2}}\right]^{2} e^{2 n}\right\}
$$

The mentioned ellipse perimeter with the first six sentences of the series is equal to:

$$
P_{M a c}=2 \pi a_{0}(1-0.187-0.026-0.008-0.003-0.0015)=20 \pi(0.774)=48.63
$$

The perimeter with the first eight sentences of the series is equal to:

$$
\begin{gathered}
P_{M a c}=2 \pi a_{0}(1-0.187-0.026-0.008-0.003-0.0015-0.0008-0.0004) \\
=20 \pi(0.772)=48.55
\end{gathered}
$$

And the perimeter with the first ten sentences of the series is equal to:

$$
\begin{aligned}
P_{M a c}= & 2 \pi a_{0}(1-0.187-0.026-0.008-0.003-0.0015-0.0008-0.0004 \\
& -0.0002-0.00015)=20 \pi(0.771)=48.53
\end{aligned}
$$

As you can see, when the number of sentences of the series increases, the Maclaurin perimeter approaches to the analytical perimeter. And it seems that finally, for the infinity number of sentences of the series, reaches to 48.47. Therefore, $m=2$ is correct choice, and therefore the final form of the analytical equation of the ellipse perimeter is as follows:

$$
P_{\text {Ellipse }}=\frac{2 \pi a_{0}}{\sqrt{2} \epsilon^{2}}
$$

\section{Elliptic integrals}

Now that we have the exact equation of ellipse perimeter, we can also calculate the value of integral 3. we have:

$$
\begin{aligned}
P_{\text {Ellipse }} & =a_{0}\left(1-\epsilon_{0}{ }^{2}\right) \int_{0}^{2 \pi} \sqrt{\frac{\left(1-\epsilon_{0} \operatorname{Cos} \theta\right)^{2}+\epsilon_{0}{ }^{2} \operatorname{Sin}^{2} \theta}{\left(1-\epsilon_{0} \operatorname{Cos} \theta\right)^{4}}} d \theta=\frac{2 \pi a_{0}}{\sqrt{2}^{\epsilon_{0}}} \\
& \Rightarrow \int_{0}^{2 \pi} \sqrt{\frac{\left(1-\epsilon_{0} \operatorname{Cos} \theta\right)^{2}+\epsilon_{0}{ }^{2} \operatorname{Sin}^{2} \theta}{\left(1-\epsilon_{0} \operatorname{Cos} \theta\right)^{4}}} d \theta=\frac{2 \pi}{\sqrt{2}^{\epsilon_{0}^{2}}\left(1-\epsilon_{0}^{2}\right)}
\end{aligned}
$$

In addition, the perimeter in Cartesian coordinates is obtained by substituting equation 10 (which is the ellipse equation in Cartesian coordinates) in 11

$$
\begin{gathered}
\frac{x^{2}}{a_{0}{ }^{2}}+\frac{y^{2}}{b_{0}{ }^{2}}=1 \Rightarrow y= \pm b_{0} \sqrt{1-\frac{x^{2}}{a_{0}{ }^{2}}} \\
P=\int_{\alpha}^{\beta} \sqrt{1+\left(\frac{d y}{d x}\right)^{2}} d x \stackrel{10}{\Rightarrow} P_{\text {Ellipse }}=\int_{0}^{2 \pi} \sqrt{1+\left(\frac{d y}{d x}\right)^{2}} d x=\int_{0}^{2 \pi} \sqrt{1+\frac{b_{0}{ }^{2} x^{2}}{a_{0}{ }^{4}\left(1-\frac{x^{2}}{a_{0}{ }^{2}}\right)}} d x
\end{gathered}
$$


Equation 11 must be equal to relation 7, therefore:

$$
\int_{0}^{2 \pi} \sqrt{1+\frac{b_{0}^{2} x^{2}}{a_{0}{ }^{4}\left(1-\frac{x^{2}}{a_{0}{ }^{2}}\right)}} d x=\frac{2 \pi a_{0}}{\sqrt{2}^{\epsilon_{0}{ }^{2}}}
$$

We obtain the values of two elliptic integrals in equations 9 and 12. Calculating these two integrals may open the way to solve elliptic integrals in terms of elementary functions. Because, as you know, so far man has not been able to write the solution of elliptic integrals in terms of elementary functions. And so far, attempts to solve elliptic integrals analytically have failed.

\section{Conclusion}

It seems that after more than 300 years, we have been able to obtain the exact equation of perimeter of a flat ellipse.

\section{References:}

[1]. Silverman, R. Calculus with Analytic Geometry (Prentice-Hall, Inc. New Jersey. 1985), pp. 628-641

[2]. Johnson, R. Calculus with Analytic Geometry (Allyn and Bacon. Inc. Boston, ed. 4), pp. 536-539

[3]. Sherman, S. Calculus with Analytic Geometry (McGraw-Hill, Inc., ed. 3, 1982), pp. 548-554

[4]. Mazer, A. The Ellipse (John Wiley \& Sons, Inc. 2010), pp. 152-154

[5]. Maclaurin, C. A Treatise of Fluxions in Two Books, vol. 2, T. W. and T. Ruddimans, Edinburgh, 1742.

[6]. www.grancorporation.com/Ellipse_Perimeter_Rev-.pdf 